\title{
La situación de la producción científica latinoamericana en el área de la Ciencia de Información Geográfica
}

\author{
The state of affairs of the latin-american GIScience \\ scientific production
}

\author{
Carlos López-Vázquez* \\ Miguel-Ángel Bernabé-Poveda**
}

Recibido 6 de enero de 2020; aceptado 30 de enero de 2020

\section{Resumen}

Se ha planteado el objetivo para la Revista Cartográfica de captar un mínimo de 45 artículos por año, con el fin de ser considerado su ingreso al catálogo SciELO. Para valorar lo razonable del objetivo se ha intentado por primera vez estimar el volumen de la producción científica de los países miembros del Instituto Panamericano de Geografía e Historia en el área de la Ciencia de la Información Geográfica. Se realizó un análisis bibliométrico de los artículos publicados en un conjunto representativo de revistas internacionales, filtrando aquellos cuyos autores son de países de la región. Se excluyó a los Estados Unidos, en razón de su peso relativo en el área. El objetivo principal es duplicar la recepción de trabajos para publicar ser publicados en la Revista Cartográfica, aunque pareciera difícil de cumplir en un corto plazo. Como alternativa, se considera interesar a autores fuera de la región, quienes típicamente toman primero en cuenta la categorización de un revista, misma que no se ha logrado con Revista Cartográfica, volviéndose un círculo vicioso de difícil solución. Subsi-

* Universidad ORT URUGUAY, Uruguay, correos electrónicos: carloslopez@uni.ort.edu.uy; carlos.lopez@ieee.org. ORCID: http://orcid.org/0000-0002-8444-1510

** Universidad Nacional de Catamarca, Argentina, correo electrónico: ma.bernabe@gmail.com. ORCID: https://orcid.org/0000-0003-4502-3524 
diariamente se ha realizado un análisis discriminado por países que puede ser utilizado en el futuro para identificar grupos y líneas activas de investigación en la región.

Palabras clave: Ciencia de la Información Geográfica, cienciometría, IPGH, SciELO, Latindex.

\begin{abstract}
The goal for Revista Cartográfica to publish a minimum of 45 articles per year has been raised in order to be considered to enter the SciELO catalog. To assess the feasibility of the objective, an estimate of the volume of scientific production of the Pan American Institute of Geography and History member countries in the area of Geographic Information Science has been attempted for the first time. A bibliometric analysis of the articles published in a representative set of international journals was performed, filtering those whose authors are from countries in the region. The US was excluded because of its high relative weight in this scientific area. The main conclusion is that the goal of capturing part of these works and thus more than duplicating the material published today in Revista Cartográfica seems difficult to achieve in the short term. It will be necessary to interest authors from outside the region, who typically first take into account the journal's categorization, a categorization that has not yet been achieved, thus forming a vicious circle of difficult solution. Subsidiarily, a country-discriminated analysis has been carried out that could be used in future works to identify active research groups and research lines in the region.
\end{abstract}

Key words: Geographic Information Science, PAIGH, SciELO, Latindex.

\title{
Introducción
}

El Instituto Panamericano de Geografía e Historia (IPGH), es un organismo gubernamental panamericano, creado en 1928 y vinculado desde 1949 con la Organización de Estados Americanos (OEA), en carácter de organismo especializado. Su actividad técnica se organiza en cuatro comisiones, a saber Cartografía (creada en 1941), Historia (1946), Geografía (1946) y Geofísica (1969). A falta de una definición expresa de su área de competencia, la Comisión de Cartografía del Instituto ha asumido que las áreas hoy consideradas dentro de la Ciencia de la Información Geográfica (CIG en lo sucesivo) serían las que al presente mejor la describiría, sin perjuicio de reconocer que hay una intersección importante pero parcial con otras ramas de la ciencia bien establecidas como la Geodesia.

Según reza su estatuto, el IPGH tiene por objeto “...Fomentar, coordinar y difundir los estudios cartográficos, geofísicos, geográficos e históricos y los relativos a las ciencias afines de interés para América...”. Este trabajo apunta a apoyar la misión de difusión, la cual en el área específica de la CIG se lleva adelante mediante la Revista Cartográfica (RCA en lo sucesivo), creada en 1952 como revista anual. Desde esos 
comienzos, y en un proceso de mejora continua, se fueron ajustando métodos y definiciones y finalmente se pasó en 2016 a dos números anuales (unos 18 a 20 artículos por año) permitiendo así que RCA sea incluida en Latindex (http://www.latindex.org). Ahora se está considerando cumplir con los requisitos para ingresar en SciELO (http://www.scielo.org), un sistema colectivo iberoamericano para publicación electrónica cooperativa de revistas científicas en Internet que tuvo su origen en Brasil. Tanto Latindex como SciELO son catálogos colectivos que recogen los nombres de publicaciones periódicas de la región que cumplen ciertas características. Básicamente se plantean requisitos de tipo editorial que, aunque no implican al contenido, obligan al editor a garantizar un grado de calidad en la operativa. Hay versiones mundiales equivalentes: por ejemplo, el Journal Citation Reports (JCR en lo sucesivo), SCOPUS, SCIMAGO, etc. Una utilidad (tal vez secundaria) de estos catálogos es ofrecer criterios de forma independiente para valorar la seriedad de una revista, y por lo tanto medir indirectamente el mérito de publicar en ellas. Eso es utilizado por los organismos de promoción de la ciencia en cada país para asignar fondos, promocionar investigadores, definir áreas prioritarias, etc. Estos organismos asignan menos puntaje por publicar en una revista de la que se ignora si tiene un Comité Editorial o si lo tiene no se conocen sus integrantes, tiene periodicidad desconocida, etc., todos aspectos muy técnicos, por lo que para su dilucidación se recurre a servicios de catalogación especializados. No es el objetivo aquí discutir los requerimientos de cada uno de estos catálogos; sí se puede decir que en la región latinoamericana se considera que Latindex es un nivel mínimo a cumplir, y que SciELO constituye un escalón superior. Una vez alcanzada esa clasificación regional, la siguiente etapa será ingresar a un catálogo internacional tipo JCR, SCOPUS, SCIMAGO o similar.

En lo sucesivo cuando se aluda a la comunidad científica de la región se estará aludiendo a la comunidad científica de la región en la temática CIG. Como se verá, esa comunidad no está claramente identificada, así como tampoco hay un criterio estricto para hacerlo. Se define la región como el conjunto de los países miembro del IPGH exceptuando Estados Unidos, por razones que se presentarán más adelante. Entre otros requisitos formales, para que una revista pueda ser considerada por SciELO, se necesita asegurar regularmente un volumen mínimo de artículos por año. Ese mínimo no es especificado centralmente, sino que cada país define cual será. En el caso de México (lugar donde se publica RCA) se ha manejado seguir el criterio de Brasil, que al presente especifica un mínimo de 45 artículos por año (recomendado 55) para revistas del área de Ciencias de la Tierra (Anon, 2017: 14). Ello es sensiblemente superior a la actual producción recogida en RCA que es de unos 20 artículos anuales repartidos en dos números. También la frecuencia debería ser modificada, recomendando publicar un número cada dos meses o como máximo tres meses, pero este aspecto no será considerado aquí. 
La tasa de rechazo de RCA es del orden del $25 \%$, en línea con lo habitual para este tipo de revistas. Lo crítico para lograr alcanzar 45 artículos anuales es asegurar un volumen superior y sostenible de trabajos enviados por los autores a su consideración. De ninguna manera se está considerando disminuir los requerimientos de aceptación. Esta investigación tuvo como propósito valorar la factibilidad de esta iniciativa en relación a los requisitos presentes de SciELO. Para ello debe estimarse el volumen total y distribución por países de la producción académica en el mundo que a) puede atribuirse al área de CIG y b) fue realizada por investigadores instalados en los Estados Miembros del IPGH. Como resultado secundario puede ofrecerse un diagnóstico del estado de situación de la CIG en la región.

Este artículo está organizado en varios apartados. En Antecedentes se analizarán trabajos similares, que en particular definen listados de revistas de interés para el área CIG. Posteriormente se discutirán los Datos y Métodos aplicados, sus limitaciones y características. A continuación se presentarán los Resultados, discriminando en producción por países y finalmente se presentarán las Conclusiones junto con un esbozo de los trabajos futuros.

\section{Antecedentes}

La delimitación temática de la CIG (nombre introducido por Goodchild, 1992) ha sido abordada por varios autores pero aún no puede decirse que sea un tema cerrado (Di Biase et al. 2006; Prager y Plewe, 2009; Kemp, Kuhn y Brox, 2013; Reitsma, 2013; Blaschke y Merschdorf, 2014; Biljecki, 2016). Las ciencias nuevas son, en muchos casos, una mezcla de temas reclasificados de otras ciencias consolidadas más la incorporación de nuevos temas surgidos indirectamente de avances tecnológicos o de otros eventos externos (Xie et al., 2017). Sin perjuicio de aceptar la existencia de revistas de ámbito general, las revistas específicas caracterizan a las ciencias establecidas (Yuan, 2017). Se asume implícitamente que el Editor y sus asesores filtran estrictamente los trabajos que se reciben de acuerdo con una línea establecida, y que esa línea además se mantiene en el tiempo. En esas hipótesis hay una asociación directa entre el área científica y un conjunto dado de revistas que cubren la temática. Debe dejarse constancia de que la irrupción de nuevos temas y soluciones, así como el desarrollo de nuevas tecnologías, para atender a viejos temas, pone en tensión ese modelo ya que, o bien hay que decidir si se extiende el ámbito temático de la revista, o bien si se aceptan trabajos de temáticas que a priori no hubieran sido de interés.

Para conocer qué revistas publican trabajos del ámbito de la CIG se analizaron varios trabajos:

- Apoyándose en expertos y siguiendo el proceso de encuestas Delphi, Caron et al. (2008) construyeron un primer listado de las revistas más importantes del área 
CIG con el fin de orientar a los investigadores a dónde remitir sus trabajos. Existían trabajos previos metodológicamente similares, pero no contemplaban específicamente el área CIG. Mayormente (pero no exclusivamente) el listado incluye revistas en idioma inglés, sin restricción expresa a un ámbito geográfico. El listado de la Tabla 1, considera 46 revistas organizadas en cuatro grupos que los autores indican son de importancia decreciente. Las revistas tienen sede en Estados Unidos, Europa Occidental y Canadá. No son necesariamente especializadas en CIG sino que su presencia en el listado debe interpretarse como que habitualmente aceptan trabajos de CIG; este detalle es de particular importancia, y será retomado luego. La utilidad de este listado es ponderar el mérito de los autores al publicar en esas revistas.

- Kemp, Kuhn y Brox (2013) elaboraron también un listado de revistas de CIG utilizando un procedimiento Delphi similar al de Caron et al. (2008). La clasificación se organizó en tres grupos: a), que contenía los títulos líderes en el área, b) y c), cada uno con menor importancia que el previo. La diferencia entre estos dos últimos era debida a cumplir o no algunas características formales (comité de evaluación internacional o no; uso de al menos dos revisiones independientes o no, etc.). La encuesta se planteó a individuos con título de $\mathrm{PhD}$ y que hubieran publicado en al menos cinco revistas diferentes del área. La misma fue contestada mayoritariamente por individuos de Europa y Estados Unidos, seguramente sesgando así los resultados. Los autores ponen énfasis en destacar que el ranking resultante no debe tomarse como definitivo sino como orientativo, a falta de mejores y más profundos trabajos que lo validen. Como elemento adicional al de revistas, los autores ofrecen un ranking de Conferencias.

- En un interesante enfoque Scarletto (2014) construyó un listado de revistas de CIG a partir de las citas encontradas en solamente cuatro de ellas en el período 2008-2010: (1) International Journal of Geographical Information Science (IJGIS en lo sucesivo), (2) Annals of the Association of American Geographers, (3) Cartography and Geographic Information Science, and (4) Cartographic Journal. Los números entre paréntesis corresponden a la categoría asignada a ellas por Caron et al. (2008). Tras restringirlo a publicaciones en inglés el resultado fue un listado de 2070 revistas. Tras analizar las citas, la autora concluye que las revistas pueden clasificarse en tres zonas, contando la más influyente con 23 títulos y la segunda con 190. El principal mérito que tiene este trabajo es que, una vez elegidas las cuatro revistas de base, el proceso para elegir los títulos más influyentes es objetivo, a diferencia del seguido por Caron et al. (2008) o Kemp, Kuhn, y Brox (2013). Desafortunadamente se descartaron revistas por no publicarse en idioma inglés, y se utilizó apenas un período de tres años para construir el ranking. El solape con el listado de Caron et al. (2008) es importante, como reconocen Melo y Queiroz (2019). 
Tabla 1

Producción acumulada y anualizada de países miembros del IPGH (excepto Estados Unidos) discriminada por revista según listado de Caron et al. (2008)

\begin{tabular}{|c|c|c|c|c|c|c|}
\hline & \multirow[t]{2}{*}{ Revista } & \multicolumn{2}{|c|}{$\begin{array}{l}\text { Promedio } \\
\text { anualizado }\end{array}$} & \multicolumn{3}{|c|}{$\begin{array}{l}\text { Acumulado hasta } \\
2019\end{array}$} \\
\hline & & $09-14$ & $14-19$ & 2009 & 2014 & 2019 \\
\hline \multirow{7}{*}{ 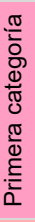 } & International Journal of Geographic Information Science & 2.0 & 4.0 & 15 & 25 & 45 \\
\hline & International Journal of Remote Sensing & 5.2 & 37.0 & 271 & 297 & 482 \\
\hline & Photogrammetric Engineering \& Remote Sensing & 2.0 & 2.2 & 24 & 34 & 45 \\
\hline & Computers and Geosciences & 7.6 & 19.6 & 86 & 124 & 222 \\
\hline & Transactions in GIS & 0.8 & 3.2 & 9 & 13 & 29 \\
\hline & Geolnformatica & 2.2 & 1.6 & 10 & 21 & 29 \\
\hline & Geomatica & 1.2 & 0.0 & 0 & 6 & 6 \\
\hline \multirow{15}{*}{ 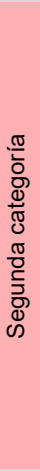 } & Cartography and Geographic Information Sciences & 0.2 & 0.8 & 2 & 3 & 7 \\
\hline & Environment and Planning B & 1.0 & 0.4 & 0 & 5 & 7 \\
\hline & IEEE Transactions Geoscience and Remote Sensing & 2.0 & 14.6 & 52 & 62 & 135 \\
\hline & Remote Sensing of Environment & 10.4 & 25.2 & 106 & 158 & 284 \\
\hline & Computers, Environment and Urban Systems & 0.8 & 2.6 & 8 & 12 & 25 \\
\hline & Annals of the Association of American Geographers & 0.2 & 1.2 & 12 & 13 & 19 \\
\hline & URISA Journal & & & & & \\
\hline & Environment and Planning $\mathrm{A}$ & 1.0 & 3.2 & 8 & 13 & 29 \\
\hline & Landscape Ecology & 5.2 & 14.2 & 29 & 55 & 126 \\
\hline & Cartographica & 0.0 & 0.4 & 1 & 1 & 3 \\
\hline & Journal of Geographic Information and Decision Analysis & & & & & \\
\hline & Marine Geodesy & 0.8 & 0.4 & 6 & 10 & 12 \\
\hline & Revue Internationale de Géomatique & & & & & \\
\hline & Journal of Geographical Systems & 0.0 & 1.4 & 0 & 0 & 7 \\
\hline & Professional Geographer & 0.6 & 1.4 & 11 & 14 & 21 \\
\hline \multirow{22}{*}{ 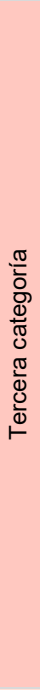 } & Transactions of the Institute of British Geographers & 0.2 & 0.4 & 1 & 2 & 4 \\
\hline & Earth Surface Processes and Landforms & 3.8 & 11.2 & 17 & 36 & 92 \\
\hline & Progress in Human Geography & 0.2 & 0.6 & 3 & 4 & 7 \\
\hline & Spatial Cognition and Computation & 0.2 & 0.8 & 3 & 4 & 8 \\
\hline & Mapping Sciences and Remote Sensing & 0.0 & 0.0 & 4 & 4 & 4 \\
\hline & Canadian Journal of Remote Sensing & 0.6 & 2.4 & 40 & 43 & 55 \\
\hline & Cartographic Journal & 0.6 & 1.6 & 1 & 4 & 12 \\
\hline & Canadian Geographer & 0.2 & 0.4 & 1 & 2 & 4 \\
\hline & Geoscience Canada & 0.0 & 0.6 & 2 & 2 & 5 \\
\hline & Cybergeo & 1.6 & 2.2 & 6 & 14 & 25 \\
\hline & International Journal of Urban and Regional Research & 2.2 & 3.2 & 29 & 40 & 56 \\
\hline & Applied Geography & 7.2 & 16.0 & 8 & 44 & 124 \\
\hline & Surveying and Land Information Science & 0.0 & 0.0 & 1 & 1 & 1 \\
\hline & Earth Observation and Remote Sensing & 0.0 & 0.0 & 0 & 0 & 0 \\
\hline & Cartographic Perspectives & 0.2 & 0.0 & 0 & 1 & 1 \\
\hline & Acta Cartographica & & & & & \\
\hline & Remote Sensing Reviews & 0.0 & 0.0 & 4 & 4 & 4 \\
\hline & Espace Géographique & 0.2 & 0.2 & 7 & 8 & 9 \\
\hline & Geofocus & & & & & \\
\hline & Mappemonde & 0.0 & 0.0 & 7 & 7 & 7 \\
\hline & Geographical Analysis & 1.0 & 1.8 & 0 & 5 & 14 \\
\hline & Geocarto International & 0.0 & 5.4 & 14 & 14 & 41 \\
\hline \multirow{3}{*}{$\geq$} & Geomatique Suisse & & & & & \\
\hline & Géospatial Solutions & 0.0 & 0.0 & 3 & 3 & 3 \\
\hline & Totales & 61.4 & 180 & 801 & 1108 & 2008 \\
\hline
\end{tabular}

Fuente: $\quad$ Elaboración propia en base a SCOPUS.

Nota: Si no hay artículos pero la revista está en SCOPUS se le indica con un cero, y se deja la celda en blanco cuando la revista no está catalogada en SCOPUS. 
- En un trabajo reciente, Biljecki (2016) hace un estudio cienciométrico (De Solla Price, 1978) específico a revistas de CIG. Del total de 20 revistas que finalmente considera, 12 están en el listado de Caron et al. (2008). En ese proceso señala varias dificultades. Una primera es que el autor considera que como aún no hay una definición precisa del ámbito de acción de la CIG ello lleva a que artículos de estas temáticas aparezcan en revistas de áreas relacionadas pero que no pueden clasificarse como "revistas de CIG". Estas incorporan trabajos de CIG pero no constituyen su ámbito exclusivo. Entre otros, los resultados presentados incluyen caracterizar la producción discriminada por país así como por revista. El autor finalmente señala que el $5 \%$ de los países son responsables del $76 \%$ de la producción científica en el área. En cuanto a la distribución por países, Estados Unidos es dominante (31.3\% del total) y de la región el único que aparece entre los primeros 15 es Brasil, con el 1.1\% del total. Finalmente, el autor presenta resultados de producción bibliográfica en relación a la población y al PBI.

Existen trabajos estrechamente relacionados con los previos, pero que ignoran la distinción entre CIG y SIG (Sistemas de Información Geográfica).

- Tian et al. (2008) realizaron un extenso trabajo cubriendo artículos que contengan "GIS" o "Geographical Information Systems" publicados en el período 19972006. La base de datos utilizada fue la Science Citation Index (SCI) suministrada por el Institute for Scientific Information (ISI) Web of Science. Los cinco países más productivos para SIG son Estados Unidos, Reino Unido, Canadá, Alemania y China; el primero tiene mucha distancia con los siguientes, por eso se ha considerado el trabajo por separado en el resto del trabajo. En relación a las revistas, señalan que el $15 \%$ del total de trabajos fueron publicados en 10 de ellas (de un total de 1918$)$, siendo IJGIS la más activa.

- Melo y Queiroz (2019) analizan artículos publicados en un conjunto de 20 revistas en el período 2006-2017, constituyendo esas 20 revistas una parte de las seleccionadas por Caron et al. (2008) y por Scarletto (2014). El criterio de selección ha sido que publiquen trabajos del área CIG o que mencionan a los SIG. Los autores confirman que la producción académica mundial del área conjunta CIG más aquellos que trabajos que usan SIG ha crecido a un ritmo sostenido de 1.8\% anual, oscilando entre 170 y 250 artículos al año. Ese valor es consistente con el reseñado por Tian et al. (2008) para el período previo 1997-2006 y con los resultados actualizados que se presentarán más adelante.

- Por último, y en un trabajo que podría considerarse como de ámbito regional (haciéndolo comparable con el presente) Li et al. (2016) analizan la producción china en cuatro revistas nacionales de topografía, geodesia, y áreas afines para el período 2003-2013. Del análisis de los 5863 artículos con participación de 
5822 autores resulta que el 59\% de los trabajos son del área Cartografía, Geodesia o SIG.

El uso de revistas específicas no es la única alternativa. Otra posibilidad sería delimitar la producción del área a través del uso de palabras clave, idea que Blaschke y Merschdorf (2014) recomiendan considerar con mucha precaución. Según ellos, los investigadores en CIG aún no han acordado una terminología precisa y los conceptos principales tal vez no están suficientemente establecidos, todo lo cual pone en duda la validez práctica de este enfoque.

\section{Datos y métodos}

En lo sucesivo se asumirá que la producción mundial en el área CIG está limitada a lo publicado en revistas del listado especificado por Caron et al. (2008). Ello es válido a los fines de acotar el volumen de producción académica regional por la que podría aspirar a competir RCA. Como un segundo criterio interesaba identificar aquellos trabajos publicados en esas revistas por autores que establecían como afiliación a alguna institución localizada en un país miembro del IPGH. Ambos criterios de búsqueda son algo arbitrarios y tienen varios inconvenientes:

- El hecho de publicar en esas revistas no implica automáticamente que la temática del artículo sea de CIG. Por lo tanto, lo que se obtendrá es una estimación optimista de la producción de la región en el tema CIG.

- No se puede valorar fácilmente la importancia de la contribución de los nacionales de los países miembros a un artículo: en algunos casos serán los principales responsables pero en casos extremos quizá aparecen como coautores simplemente por haber suministrado los datos para el artículo.

- Un tercer inconveniente, que quedará claro al discriminar la producción por países de la región, es que trabajos con autores de dos o más diferentes países miembros serán contabilizados tantas veces como corresponda, lo que exagera la producción conjunta. En contrapartida, es habitual que autores de la región realicen estudios en países de fuera de ella y publiquen con la filiación accidental que posean, siendo invisible su relación (si la hubiera) con el país de origen y por tanto reduciendo la producción regional. Se asumirá que ambas desviaciones se compensan mutuamente.

- Por último, debe señalarse que para asignar a un país el mérito de una publicación hay dos criterios. Podría usarse la última filiación conocida del autor, y atribuir a ese país toda la producción del mismo. Ello reconocería que la capacidad de producción científica está con el autor. Otro criterio es razonar que la publicación se dio en un cierto contexto, con recursos aportados por el país y que de alguna forma 
los mide. En ese caso la filiación se mantiene tal cual aparece en la publicación original, y es el criterio que se adoptará aquí.

Las revistas fueron identificadas a través de su(s) ISSN. La información se extrajo de la base SCOPUS, la cual es muy extensa pero al presente (pasados más de 10 años) todavía no incluye una media docena de las revistas del listado de Caron et al. (2008). Entre las ausentes en particular interesa vivamente el caso de Geofocus International Review of GI Science and Technology, la cual a la fecha (2019) sí está incluida en el listado de Journal Citation Reports (JCR).

Geofocus acepta artículos en castellano, por lo que su ausencia seguramente tiene un impacto no despreciable en las estimaciones de la producción regional en CIG a realizar en este trabajo. Esta revista, que tiene una media de 18 artículos por año divididos en dos números anuales, que la mayoría de sus artículos están en castellano y que, como se ha anotado antes, está incluida en JCR pero no en SciELO, es potencialmente un competidor importante para RCA. Los autores que podrían publicar en castellano en RCA (que además acepta trabajos en inglés, francés y portugués) probablemente prefieran considerar primero hacerlo en Geofocus al estar catalogada en un listado de prestigio como JCR.

En su artículo, Caron et al. (2008) clasificaron las revistas en cuatro categorías de acuerdo a su relevancia académica, reflejada en los colores de la Tabla 1. Puesto que las conclusiones de este trabajo se extraerán de lo publicado en ese conjunto de revistas, es importante comentar lo dispar del volumen publicado en alguna de ellas. Por ejemplo, en la Tabla 1 puede verse que los autores de la región publicaron en International Journal of Remote Sensing 10 veces más de lo que hicieron en IJGIS. Si se asume que la primera no es una revista especializada en CIG pero la segunda sí, la consecuencia de considerar conjuntamente lo publicado en ambas es que la componente CIG se diluye irremediablemente. Lo mismo ocurre con otras revistas de interés más general: Remote Sensing of Environment recibió más de seis veces artículos de autores regionales que IJGIS.

\section{Caracterización de la producción regional}

La caracterización de la capacidad productiva de la región en el área CIG puede hacerse de tres maneras:

1. Identificando los autores CIG de la región y caracterizando su producción en cualquier revista o,

2. localizando los artículos CIG basado en su contenido pero publicados en cualquier revista y reteniendo aquellos con autores de la región o,

3. identificando un conjunto de revistas representativas y analizando la producción con autores de la región. Este fue el procedimiento finalmente seguido. 
Aunque las alternativas (a) y (b) no son de implementación directa se discutirán más adelante. La consulta a SCOPUS se realizó en diciembre de 2019 de dos maneras:

- Para cada revista del listado, se contabilizó el número de trabajos con al menos un autor cuya filiación estuviera en alguno de los países miembros. Dado el (los) ISSN correspondientes de cada revista, manualmente se descargó un fichero en formato CSV y el resto del procesamiento se hizo localmente.

- Para un país miembro, se contabilizó el número de trabajos en cualquiera de las revistas del listado con al menos un autor con afiliación en ese país. Como se encontraron algunas inconsistencias con los datos previos se descartó utilizarlos.

El uso de un banco de datos dinámico como SCOPUS tiene sus dificultades, porque tras reiterar más tarde las mismas consultas algunos resultados no son repetibles. Se notó también que hubo revistas del listado que inicialmente no aparecían, luego aparecían y finalmente desaparecían nuevamente tiempo más tarde, por lo que se optó por utilizar únicamente la primera de las consultas mencionadas, e inferir de los datos descargados todo lo demás.

\section{Identificación de países}

Tanto SCOPUS como otras bases del mismo tipo ocasionalmente tienen problemas con la afiliación de los autores; la construcción de estos servicios rescata del pasado analógico datos para su procesamiento en el presente, y el proceso de digitalización tiene sus debilidades. Puede verse que por ejemplo muchos trabajos de las universidades de Estados Unidos simplemente no declaran explícitamente el país. También ocurre con algunas universidades europeas y con muchas empresas y organismos oficiales de investigación. Otros problemas son las diferentes formas de codificar los nombres: en ocasiones en los registros figuran abreviaturas como "Engl" y en otras "England". Por lo tanto el volumen de producción por país debe ser considerado como conservador, pudiendo haber más trabajos que no fueron correctamente imputados.

\section{Identificación de autores}

Otro problema no menor es la dificultad que existe en asignar correctamente la autoría a un autor basado simplemente en el nombre, problema ya reconocido por Sun y Mason (2001) también trabajando en el área de CIG. Hasta hace muy poco tiempo no existía alternativa para bases como SCOPUS más que intentar interpretar el nombre (con sus abreviaturas) y asignarle a un único identificador la autoría de varios trabajos considerando a la vez la afiliación, tal vez la temática, etc. 
El proceso funciona bien para nombres y apellidos infrecuentes, pero necesariamente falla en otros casos. Ello se agrava con las ambigüedades en la forma de auto-denominarse, que pueden cambiar con el paso del tiempo. La situación a futuro va en vías a mejorar drásticamente, en la medida que se populariza el uso de identificadores personales como ORCID (www.orcid.org) que resuelven estas ambigüedades (Lane, 2010; Haak et al., 2012).

\section{Producción por país}

La estadística de producción por autor, institución, país, etc. puede hacerse de varias formas (Biljecki, 2016):

- En una, se cuentan las apariciones de un autor, en particular en los artículos publicados, independientemente del número de coautores. Ello tiene el inconveniente de que ciertos colectivos que acuerdan publicar conjuntamente están sobrerrepresentados en el total. Una primera contramedida para ello es contabilizar únicamente a los autores que están en primer lugar, bajo la hipótesis de que ese puesto se reserva para el autor más importante. No siempre es así, y en ciertos trabajos colectivos se señala explícitamente que el listado de autores está en orden alfabético. Aunque se especifique dentro del cuerpo del artículo, SCOPUS no lo contabiliza.

- Otro criterio es ponderar la aparición de un autor en particular con el inverso del número de coautores. La ventaja de este enfoque es que la suma de la contribución asignada a todos los autores iguala al número de artículos, pero igual que antes ignora cualquier diferencia entre los aportes de cada autor.

- Aún pueden combinarse los criterios anteriores: Kumar y Khormi (2012), asignaban un peso diferente entre el 0.75 para el primer autor y 0.25 para repartir equitativamente con el resto.

En el trabajo que aquí se presenta se ha ponderado el aporte a cada país teniendo en cuenta el inverso del número de coautores.

\section{Resultados}

\section{Publicaciones de la región}

La Figura 1 muestra para el período 1995-2020 simultáneamente la producción anual y la acumulada de los países miembros del IPGH excluyendo a Estados Unidos. Por razones prácticas la búsqueda separó a ese país, ya que si bien pertenece al IPGH tiene un volumen de publicaciones que excede al menos un orden de magnitud (10 veces) el del resto de países del IPGH. Por los errores sistemáticos ya mencionados 
ésto es incluso un valor conservador, ya que hay muchos trabajos que siendo de Estados Unidos no fueron correctamente imputados a ese país. Independientemente de ello, y para los objetivos de este artículo, la consideración de incluir a dicho país, impediría valorar adecuadamente la situación de la región.

La evolución reciente de la situación en la región que se exhibe en la Figura 1 puede considerarse como positiva. Hay un sostenido aumento de la producción anual, que en 2011 excedió por primera vez los 100 trabajos y que actualmente alcanzó un pico de 150 por año. Por las razones ya expuestas no está claro qué parte de esa producción puede clasificarse como $\mathrm{CIG}$, pero de igual manera el resultado sirve como cota superior. El aporte total acumulado de la región ha evolucionado fuertemente en el período, desde 99 artículos publicados hasta 1995 a cerca de 2000 al presente. El crecimiento es más que lineal, lo que puede interpretarse como, que la comunidad está efectivamente en un proceso de expansión.

Para contrastar, se ha construido un gráfico equivalente (Figura 2) para la producción académica mundial anual en el mismo conjunto de revistas especificado. La curva muestra un crecimiento más próximo a lineal, correspondiente a una comunidad más consolidada. La producción es del orden de los 4000 artículos por año, por lo que la región con 150 artículos en el mismo periodo año contribuye con menos del $4 \%$ del total.

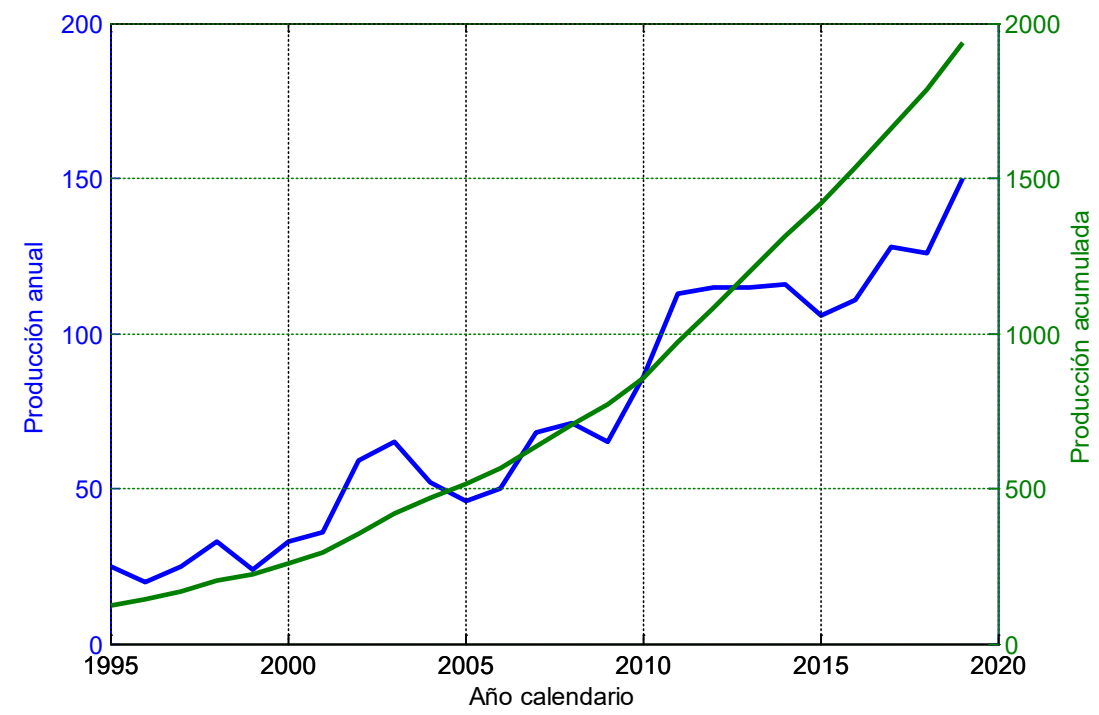

Figura 1 Producción anual y acumulada en el área CIG para países miembros del IPGH (a excepción de Estados Unidos). 


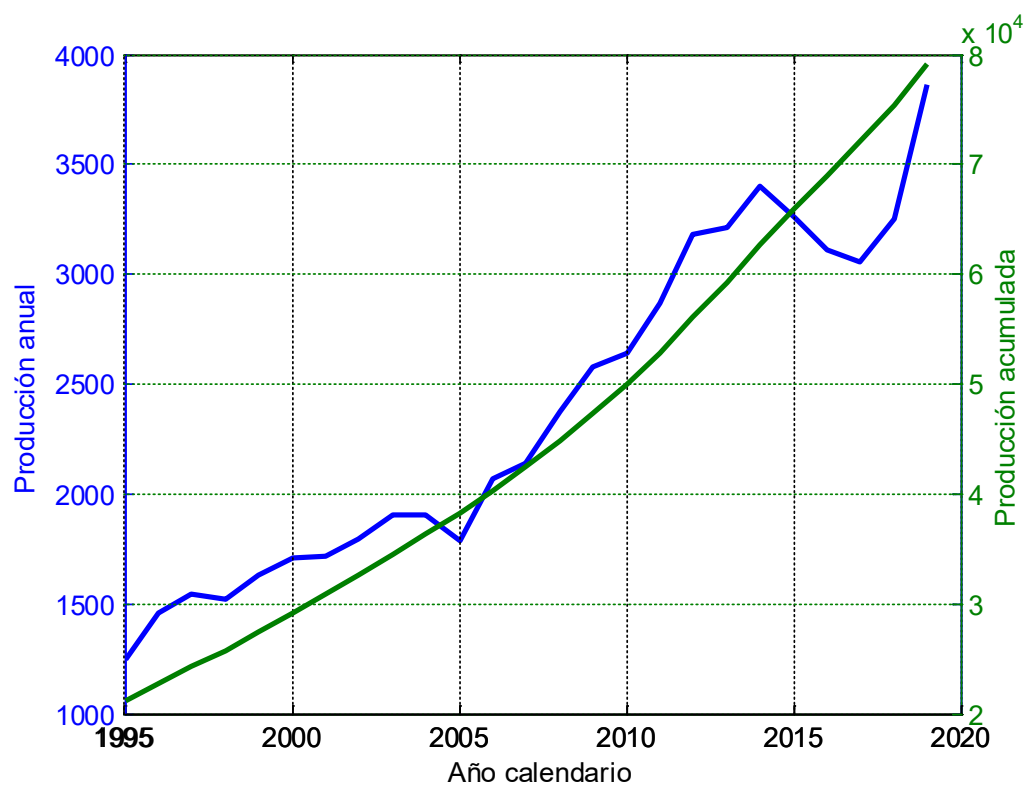

Figura 2 Producción mundial, anual y acumulada, en las mismas revistas consideradas en la Figura 1.

\section{Posible valoración de países}

Los méritos relativos de cada país/autor/institución debido a sus publicaciones tomarán la forma de un puntaje, y los países con mayor puntaje son los más destacados. El problema es elegir la forma de calcular el puntaje, para lo que en este trabajo se han usado dos alternativas:

- Para los gráficos, se han contado simplemente el número de artículos.

- Para las tablas que se presentan a cada país se le asigna un puntaje por cada artículo publicado equivalente a $1 / \mathrm{n}$, siendo $n$ el número de coautores del mismo.

En la Tabla 2 se presenta el resultado para la región. La producción por año calendario muestra demasiadas variaciones, por lo que se presentan resultados anualizados obtenidos para dos quinquenios sucesivos.

Con base en la información de la Tabla 2, en la Tabla 3 se han organizado bloques de países. Con el paso del tiempo Brasil sigue siendo responsable de cerca de la mitad de la producción regional, pero ha caído el peso relativo de los siguientes tres productores grandes y aumentado el del resto. No se presenta aquí, pero la contabilidad 
directa sin ponderar por el número de coautores no arroja resultados muy diferentes, ya que típicamente los números se dividen entre tres sugiriendo una proporción entre autores locales y externos. Parece ser que esta manera de repartir disminuye mucho el mérito del grupo de los tres que le siguen a Brasil (que tal vez están mejor conectados y colaboran mucho con extranjeros) mientras que aumenta levemente el mérito de Brasil y bastante el todos los demás, ya que publican con menos autores de extrazona.

\section{Tabla 2}

Producción acumulada y anualizada discriminada por países miembros del IPGH (exceptuando a Estados Unidos)

\begin{tabular}{lrrrrr}
\hline & \multicolumn{3}{c}{ Promedio anualizado } & \multicolumn{3}{c}{ Acumulado hasta 2019} \\
\cline { 2 - 6 } & $2009-2014$ & $2014-2019$ & 2009 & 2014 & 2019 \\
\hline Paraguay (PY) & 0.00 & 0.00 & 0.09 & 0.09 & 0.09 \\
Belize (BZ) & 0.00 & 0.07 & 0.00 & 0.00 & 0.33 \\
Guatemala (GT) & 0.00 & 0.00 & 0.31 & 0.31 & 0.31 \\
Haití (HT) & 0.00 & 0.00 & 0.50 & 0.50 & 0.50 \\
República Dominicana (DO) & 0.00 & 0.03 & 1.08 & 1.08 & 1.25 \\
El Salvador (SV) & 0.05 & 0.07 & 0.50 & 0.75 & 1.12 \\
Nicaragua (NI) & 0.00 & 0.09 & 0.44 & 0.44 & 0.89 \\
Honduras (HN) & 0.03 & 0.07 & 0.14 & 0.31 & 0.64 \\
Uruguay (UY) & 0.46 & 0.28 & 4.46 & 6.75 & 8.17 \\
Bolivia (BO) & 0.17 & 0.19 & 3.25 & 4.08 & 5.02 \\
Panamá (PA) & 0.17 & 0.51 & 0.71 & 1.57 & 4.10 \\
Venezuela (VE) & 0.13 & 0.22 & 4.98 & 5.63 & 6.74 \\
Costa Rica (CR) & 0.43 & 0.06 & 6.04 & 8.17 & 8.45 \\
Perú (PE) & 0.40 & 1.10 & 2.08 & 4.09 & 9.58 \\
Ecuador (EC) & 0.46 & 0.89 & 4.16 & 6.45 & 10.89 \\
Colombia (CO) & 1.82 & 2.92 & 13.85 & 22.94 & 37.53 \\
Chile (CH) & 5.55 & 4.75 & 26.63 & 54.36 & 78.12 \\
Argentina (AR) & 3.16 & 3.21 & 37.87 & 53.69 & 69.77 \\
México (MX) & 6.34 & 4.97 & 57.41 & 89.12 & 113.95 \\
Brasil (BR) & 14.91 & 17.66 & 149.89 & 224.46 & 312.77 \\
\hline \multicolumn{1}{c}{ Total IPGH } & 34.08 & 37.08 & 314.38 & 484.80 & 670.21 \\
(exeptuando Estados Unidos) & & & & &
\end{tabular}

Fuente: Elaboración propia en base a SCOPUS.

Nota: El conteo es ponderado, agregando a cada país una cuotaparte de la unidad teniendo en cuenta el número de coautores. 
Tabla 3

Producción anualizada y organizada por grupos, expresados en porcentaje a la producción total de la región

\begin{tabular}{lcccrc}
\hline & \multicolumn{4}{c}{ Promedio anualizado } & \multicolumn{3}{c}{ Acumulado hasta 2019 } \\
\cline { 2 - 6 } & $2009-2014$ & $2014-2019$ & 2009 & 2014 & 2019 \\
\hline BZ, DO, GT, HT, HN, SV, NI y PY & 0.24 & 0.89 & 3.06 & 3.48 & 5.13 \\
BO, CO, CR, EC, PE, PA, UY y VE & 11.83 & 16.61 & 39.53 & 59.68 & 90.48 \\
AR, CH y MX & 44.17 & 34.87 & 21.91 & 197.17 & 261.84 \\
BR & 43.76 & 47.63 & 149.89 & 224.46 & 312.77 \\
\hline
\end{tabular}

Fuente: Elaboración propia en base a SCOPUS.

\section{Análisis y discusión}

Hay varias observaciones que pueden hacerse con las diferentes tablas y figuras presentadas.

\section{Producción}

De la Figura 1, puede inferirse que, con fuertes oscilaciones año con año, hay una sostenida aceleración (excediendo la aproximación lineal) en la producción global de la región que recién alcanzó en 2019 un pico histórico de 150 trabajos anuales. Estos resultados están repartidos muy desigualmente en términos de países. Las Tablas 2 y 3 , que recogen los valores totales por país acumulados hasta el año de referencia, muestra que en la región (definida como IPGH sin Estados Unidos):

- $\quad$ BR ha publicado casi la mitad del total (46.7\%)

- Tres países (AR, $\mathrm{CH}$ y MX) colectivamente han aportado un 39.0\%

- Ocho países (BO, CO, CR, EC, PE, PA, UY y VE) cerca del 13.5\%

- Otros ocho países (BZ, DO, GT, HT, HN, SV, NI y PY) el 0.8\%

A modo de referencia en la Tabla 4 se hace una comparación internacional, en la que puede verse que, globalmente, cada cinco años hubo un $61 \%$ más de producción. En este caso se contaron simplemente artículos, sin penalizar por el número de coautores. Para darle perspectiva a este resultado debe notarse que España sola es sistemáticamente más que toda Iberoamérica, y a su vez Francia casi triplica a esa producción. Canadá casi cuadruplica la producción de la región, mientras se confirma que Estados Unidos genera del orden de 10 veces lo de la región. 
Como notables desempeños en el período pueden señalarse el grupo de Ecuador y Colombia que han venido aumentando su ya importante presencia en cada quinquenio. Se puede agregar Panamá al listado de los que duplican, pero con menos peso porque su producción absoluta es menor.

Tabla 4

Producción acumulada y anualizada de países del IPGH (sin Estados Unidos) como bloque y comparación con España, Francia, Canadá y Estados Unidos

\begin{tabular}{lrrrrr}
\hline & \multicolumn{2}{c}{ Promedio anualizado } & \multicolumn{3}{c}{ Acumulados hasta este año } \\
\hline & $2009-2014$ & $2014-2019$ & 2009 & 2014 & 2019 \\
\hline IPGH sin & & & & & \\
Estados Unidos & 108.8 & 133.4 & 781 & 1325 & 1992 \\
España & 134.8 & 167.2 & 792 & 1466 & 2302 \\
Francia & 217.4 & 273.4 & 2725 & 3812 & 5179 \\
Canadá & 314.6 & 377.0 & 5419 & 6992 & 8877 \\
Estados Unidos & 1069.8 & 1340.4 & 18275 & 23624 & 30326 \\
\hline
\end{tabular}

Fuente: Elaboración propia en base a SCOPUS.

\section{Multiautoría}

Debe recordarse que la metodología seguida impide ver detalles; la mera presencia de un único autor con afiliación en un país ya define un aporte. Con esos datos se podría haber construido tablas de autores, instituciones o países, pero dados los objetivos de este trabajo solamente se presentan discriminadas por países. Los resultados exhibidos dependen de la forma de valorar los méritos. No se presentan aquí, pero si se contabiliza simplemente el número de artículos hay un dominio importante de autores de Brasil, seguido muy de lejos por México. La existencia de grupos grandes (tanto de coautores como alumnos) parece tener un efecto importante en este resultado. $\mathrm{Si}$, con el fin de filtrar ese efecto, se contabiliza en cambio el número de artículos del país del primer autor, la tabla tiene también varios cambios.

\section{Publicaciones de CIG en revistas no especializadas}

Algunas revistas del listado tienen un volumen de artículos mucho mayor que el resto. El área CIG no es tan activa como para que aporte miles de artículos al año, como parecieran sugerir estas estadísticas mundiales. Con algunas escasas excepciones (tal vez aquellas revistas que en su título mencionan CIG expresamente) en el resto y tras analizar algo más detenidamente los contenidos puede verse que es difícil sostener que por haber sido publicado allí un artículo trate siempre sobre CIG. El objetivo de 
Caron et al. (2008) era sugerir revistas apropiadas para publicar sobre CIG, pero en general ello no implicaba una especialización temática de las mismas sino apenas una adecuada receptividad.

\section{Futuro}

Si bien en este trabajo se ha usado un listado prefijado de revistas, en futuros trabajos se podrían considerar al menos dos alternativas, no exentas de problemas:

- La más simple sería identificar primero a los autores y luego rastrear su producción en cualquier revista. Además de la dificultad en identificar por anticipado a los autores, este enfoque presume que a lo largo de su vida útil el autor se mantendrá siempre apegado al área CIG cosa que no es cierta.

- Otra posibilidad es clasificar a los artículos de CIG a través de su contenido, independientemente de la revista y del autor, buscando relaciones basadas en algún tipo de palabras clave. SCOPUS dispone como atributo las palabras suministradas por el autor, pero también otras que se generan automáticamente. Esta sería la línea más promisoria si se decidiera cambiar la actual alternativa, faltando especificar el criterio de búsqueda y detalles asociados.

\section{Nuevos aspectos a tener en cuenta}

Si bien el objetivo de este trabajo no era establecer distinciones entre países, además de la producción fue posible explorar aspectos adicionales como la productividad académica per cápita o relacionarla con la inversión en investigación. Para ello se cuenta con información secundaria que se puede utilizar.

En la Figura 3 se relacionó la producción en relación al PBI y la población. En ella pueden distinguirse tres bandas: la de países con menor producción absoluta en la Tabla 2 se identifican aquí directamente con países que tienen simultáneamente un bajo PBI/habitante y una baja productividad en términos de artículos/PBI educativo. En estos casos la baja producción (medida por el número de artículos) ocurre simultáneamente con una baja productividad (medida como el cociente entre número de artículos y otra magnitud, como el número de habitantes o el PBI). En la Figura 3 se distingue luego una banda central, en la que coexisten países de gran tamaño y producción (Brasil, Argentina y México) junto con otros de tamaño medio (Venezuela, Perú y Colombia) así como algunos más pequeños (Costa Rica y Ecuador). Fuera de esta segunda región se encuentra Belice (con una productividad aparentemente alta pero asociada con un puñado de artículos), Chile y Panamá (que tienen alto $\mathrm{PBI} /$ habitante y además una alta productividad) y Uruguay, con alto $\mathrm{PBI} /$ habitante 
pero relativamente baja productividad. Los datos corresponden a la producción ponderada y promediada del último quinquenio, mientras que los del PBI son de 2018 (excepto para Venezuela, para el que se usó el último dato disponible de 2014).

\section{Conclusiones}

Si se deseara cuantificar la producción de la región en temas de CIG, la estrategia de contabilizar simplemente artículos publicados en un listado de revistas tiene limitaciones metodológicas importantes ya que la hipótesis de que esas revistas tocan exclusivamente temas de CIG no se sostiene. No hay estudios sistemáticos al respecto, y entre otras razones puede argumentarse que la propia área no está rigurosamente delimitada.

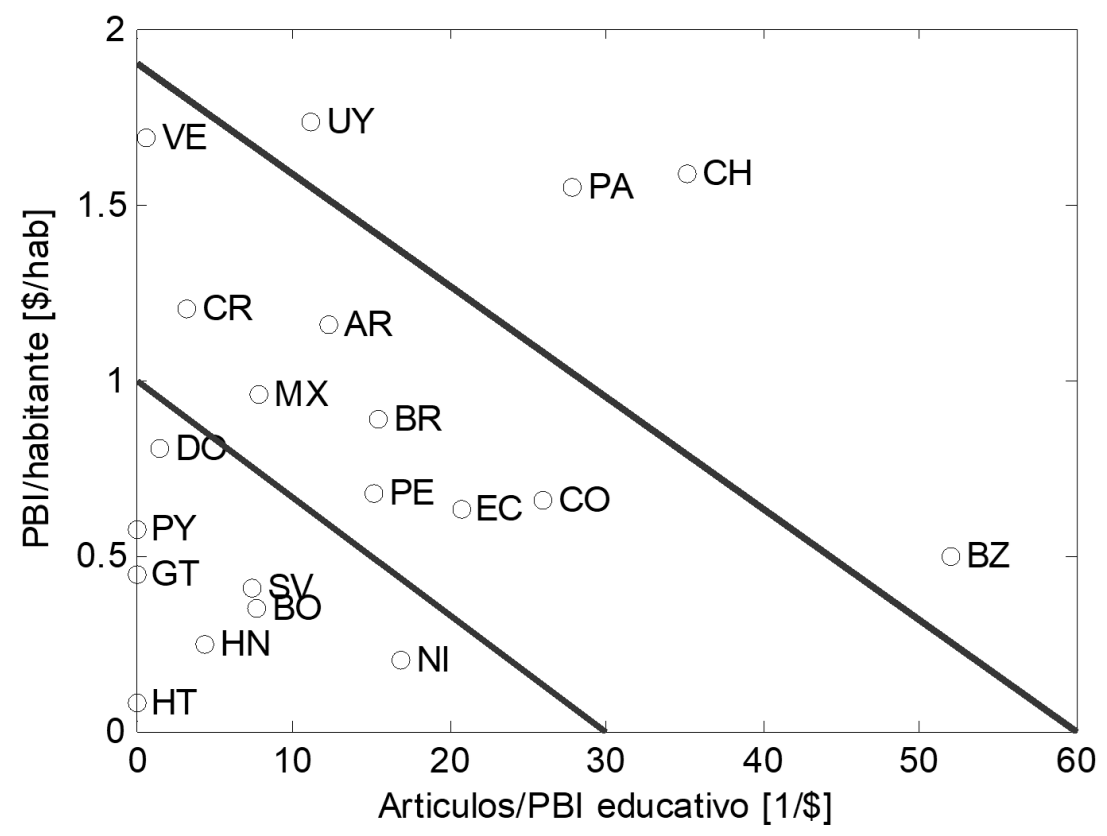

Figura 3. Productividad de los países en relación a la inversión educativa y al PBI por habitante.

Sin embargo, pueden establecerse relaciones que permiten dar cotas superiores para los valores de interés. La producción de España en ese listado de revistas es del orden de 170 artículos por año, mientras que lo publicado en GeoFocus (revista española especializada en el área) está en el eje de los 20 artículos también por año, constituyéndose en un $20 /(170+20)^{*} 100=10 \%$ del total. Si se asume que el ámbito natural de operación de RCA está en los países miembro del IPGH puede establecerse 
un paralelismo con la situación de GeoFocus, quien estando especializada en CIG capta un 10\% del volumen de las revistas del listado. La producción de toda la región IPGH, calculada en las mismas condiciones, es de 134 artículos por año (en promedio). Si se admite que la parte de CIG es del orden del 10\% del total, entonces se concluye que la fracción de CIG generada en la región sería de 13 a 14 artículos al año, que sumado a lo hoy publicado por RCA queda aún muy por debajo de lo requerido por SciELO. El cálculo previo es muy aproximado, y tiene carencias metodológicas ya expresadas. Además no hay que olvidar que RCA hoy no tiene el atractivo que tiene GeoFocus, que ya está catalogada en JCR.

La hipótesis de que los trabajos de CIG se publican únicamente en un listado de revistas especializadas no se sostiene. Una alternativa posible sería identificar autores de la región que trabajen en área CIG, y rastrear sus publicaciones personales en un conjunto abierto de revistas. Esto tiene el inconveniente de requerir la generación de la lista inicial de nombres, y un problema de fondo debido al hecho de que un investigador puede eventualmente entrar o salir del área temática sin previo aviso.

Como otra posibilidad, y siempre para futuros trabajos, se podría intentar determinar la pertenencia al área CIG no a nivel de revista sino de artículo, lo que una vez establecidos los criterios objetivos para ello llevará necesariamente a expandir la búsqueda fuera de un listado cerrado de revistas. Las alternativas que se imaginan para ello incluyen construir y utilizar un conjunto de palabras clave (propuestas por cada autor o generadas por SCOPUS), o siguiendo a Scarletto (2014) construir el conjunto con los trabajos que citan uno o varios emblemáticos que hayan sido previamente declarados como de CIG.

Puesto que el IPGH, como asociación de estados nacionales incluye prácticamente a toda América (con la excepción notable de Canadá, Cuba, Jamaica y los pequeños países del Caribe) puede considerarse que las estadísticas realizadas (en las que se excluyó deliberadamente a Estados Unidos a pesar de ser miembro) son representativas del total ya que la producción acumulada de Cuba y Jamaica hasta diciembre de 2019 era de apenas 12 y 14 trabajos respectivamente, mientras que el resto de la zona Caribe aportaba solamente 9 trabajos.

El desafío de incluir RCA en SciELO logrando los 45 artículos por año que el índice considera cantidad mínima, más que duplicando así el actual volumen debe descartarse una vez que se ha mostrado que el volumen de producción de toda la región en las revistas especializadas en CIG de más prestigio (y aun incluyendo a varias no tan prestigiosas) es del orden de 134 artículos por año. No es realista aspirar a captar 25 trabajos de esa producción de alto nivel, adicionales a los 20 ya publicados anualmente, con una revista que no aparece aún en catálogos de excelencia internacionales como SciELO, SCOPUS o JCR. Como ventaja frente a otras revistas internacionales puede argumentarse la flexibilidad de RCA, que recibe trabajos en cuatro idiomas incluyendo el castellano, pero no es la única revista del área CIG que lo hace. 
Si se excluye a Estados Unidos, la comunidad latinoamericana activa en CIG no es hoy tan grande como para aportar en forma sostenida suficiente material para satisfacer ese volumen de producción. Basado simplemente en el crecimiento vegetativo, sin otras medidas de promoción ni cambios en las prioridades académicas, podría esperarse alcanzar ese volumen recién para 2050.

\section{Bibliografía}

Anónimo (2017). “Criterios, política y procedimientos para la admisión y la permanencia de revistas científicas en la Colección SciELO Brasil”, Scientific Electronic Library Online, $31 \mathrm{pp}$. Recuperado de https://tinyurl.com/uc7mdyp

Biljecki, F. (2016). "A scientometric analysis of selected GIScience journals", International Journal of Geographical Information Science, 30(7): 1302-1335. https://doi.org/10.1080/13658816.2015.1130831

Blaschke, T. and Merschdorf, H. (2014). "Geographic information science as a multidisciplinary and multiparadigmatic field", Cartography and Geographic Information Science, 41(3): 196-213. https://doi.org/10.1080/15230406.2014.905755

Caron, C.; Goyer, D., Roche, S. and Jaton, A. (2008). "GIScience journals ranking and evaluation: an international Delphi study", Transactions in GIS, 12(3): 293321. https://doi.org/10.1111/j.1467-9671.2008.01106.x

De Solla Price, D. (1978). Scientometrics, 1(1): 3-8, Editorial statements.

Di Biase, D.; DeMers, M., Johnson, A., Kemp, K., Taylor Luck, A., Plewe, B. and Wentz, E. (eds) (2006). Geographic information science and technology body of knowledge. Washington, D.C., Association of American Geographers, 174 pp.

Glänzel, W., \& Schubert, A. (1985)."Price distribution: An exact formulation of Price's 'square root law'", Scientometrics, 7(3-6): 211-219.

https://doi.org/10.1007/BF02017147

Goodchild, M.F. (1992) “Geographical information science”, International Journal of Geographical Information Systems, 6: 31-45.

https://doi.org/10.1080/02693799208901893

Haak, L.L.; Fenner, M., Paglione, L., Pentz, E. and Ratner, H. (2012) "ORCID: a system to uniquely identify researchers", Learned Publishing, 25: 259-264. https://doi.org/10.1087/20120404

Kemp, K., Kuhn, W. and Brox, C. (2013). "Results of a survey to rate GIScience publication outlets", Technical report, $8 \mathrm{pp}$.

Kumar, L. and Khormi, H.M. (2012). "Hotspots, research productivity and collaboration networks in remote sensing and GIS in Australia from 1991 to 2010", Journal of Spatial Science, 57(1): 101-113.

Lane, J. (2010). "Let's make science metrics more scientific", Nature, 464: 488-489. https://doi.org/10.1080/14498596.2012.686360 
Li, J., Liu, H., Guo, W. and Yu, A. (2016). "Visual analysis based on the data of Chinese surveying and mapping journals", ISPRS Annals of Photogrammetry, Remote Sensing and Spatial Information Sciences, III(4): 41-48.

https://doi.org/10.5194/isprs-annals-III-4-41-2016

Melo, A.V.F. and Queiroz, A.P. (2019). "Bibliometric Mapping of Papers on Geographical Information Systems (2007-2016)", Bulletin of Geodetic Sciences, 25(3): e20190015. https://doi.org/10.1590/s1982-21702019000300015

Prager, S.D. and Plewe, B. (2009). "Assessment and Evaluation of GIScience Curriculum using the Geographic Information Science and Technology Body of Knowledge", Journal of Geography in Higher Education, 33:S1, S46-S69. https://doi.org/10.1080/03098260903034012

Price, D.J. de Solla (1971). Little Science-Big Science, Columbia University Press, New York and London, 4th ed., 118 pp.

Reitsma, F. (2013). "Revisiting the 'Is GIScience a science?' debate (or quite possibly scientific gerrymandering)", International Journal of Geographical Information Science, 27(2): 211-221. https://doi.org/10.1080/13658816.2012.674529

Scarletto, E.A. (2014). "Mapping the Literature of GIS", College \& Research Libraries, 75(2): 179-201.

Sun, S. and Manson, S.M. (2011). "Social network analysis of the academic GIScience community", The Professional Geographer, 63: 18-33. https://doi.org/10.5860/crl12-389

Tian, Y.G., Wen, C. and Hong, S. (2008). "Global scientific production on GIS research by bibliometric analysis from 1997-2006”, Journal of Informetrics, 2: 65-74. https://doi.org/10.1016/j.joi.2007.10.001

Xie, Y.; Efteliogl, E.; Ali, R.Y.; Tang, X.; Li, Y.; Doshi, R. and Shekhar, S. (2017). "Transdisciplinary Foundations of Geospatial Data Science", ISPRS International Journal of Geo-Information, 6(12), 24 pp. https://doi.org/10.3390/ijgi6120395

Yuan, M. (2017). "30 years of IJGIS: the changing landscape of geographical information science and the road ahead", International Journal of Geographical Information Science, 31(3): 425-434. https://doi.org/10.1080/13658816.2016.1236928 
